\title{
A Barrett's esophagus registry of over 1000 patients from a specialist center highlights greater risk of progression than population-based registries and high risk of low grade dysplasia
}

\author{
S. L. Picardo, ${ }^{1}$ M. P. O’Brien, ${ }^{1}$ R. Feighery, ${ }^{1}$ D. O’Toole,${ }^{2}$ N. Ravi, ${ }^{1}$ N. J. O'Farrell, ${ }^{1}$ J. N. O’Sullivan, ${ }^{1}$ \\ J. V. Reynolds ${ }^{1}$ \\ Departments of ${ }^{1}$ Surgery and ${ }^{2}$ Medicine, Trinity Center for Health Sciences, St. James's Hospital and Trinity \\ College Dublin, Dublin, Ireland
}

\begin{abstract}
SUMMARY. Barrett's esophagus (BE) arising from chronic gastro-oesophageal reflux (GERD) is the main pathologic precursor of esophageal adenocarcinoma (EAC). The risk of progression to high-grade dysplasia (HGD) and EAC is unclear, and recent population studies from Denmark and Northern Ireland suggest that this has been overestimated in the past. No data exist from the Republic of Ireland. A detailed clinical, endoscopic, and pathologic database was established in one center as a proposed pilot for a national registry, and initial and follow-up data were abstracted by a data manager. One thousand ninety-three patients were registered, 60 patients with HGD were excluded, leaving 1033, with a median age of 59 and 2:1 male to female ratio, and 3599 person-years of follow-up. The overall incidence of HGD/EAC was $1.33 \%$ per year overall, $0.85 \%$ if the first year is excluded. Within the first year after index endoscopy, 18 cases of HGD or EAC were identified, and 30 following the first year. Low-grade dysplasia (LGD) on index endoscopy was associated with an incidence of progression of $6.5 \%$ per year, and $3.1 \%$ when tertiary referrals were excluded. These data provide important demographic and clinical information on the population of Irish patients with BE, with incidence rates of progression higher than recently published population-based registry series, perhaps relating to sampling and pathological assessment. Low-grade dysplasia on initial biopsy is a significant proxy marker of risk of progression.
\end{abstract}

KEY WORDS: Barrett's esophagus, esophageal adenocarcinoma, high-grade dysplasia, low-grade dysplasia.

\section{INTRODUCTION}

Disease registries provide a valuable resource in tracking clinical and epidemiological data in a population. Cancer and precancerous conditions are examples, as well as chronic diseases such as diabetes mellitus. ${ }^{1}$ Registries on a large scale can help to provide information on characteristics of patients who have the disease but can also identify subgroups of patients who are at risk of progression or increased morbidity or mortality - the more comprehensive the data collected in the registry, the more information

Address correspondence to: Professor John V. Reynolds, FRCSI, Department of Surgery, Trinity Center for Health Sciences,

St. James's Hospital and Trinity College Dublin, Dublin 8, Ireland. Email: reynoljv@tcd.ie

Supported in part by the Oesophageal Cancer Fund, the Dublin

Center for Clinical Research, and Mr. Terry Stewart.

Conflict of interest:

The authors have no conflict of interest.

C 2014 International Society for Diseases of the Esophagus on risk factors and disease epidemiology provided. In general, the compiling of information for disease registries is found to be acceptable to the general public and not an invasion of privacy. ${ }^{2}$

Precancerous conditions are an obvious target for a disease registry - the data collected may help to identify patients at risk of progression to cancer, especially if the condition is common in the population being targeted. The larger the number of people on the registry, the more likely the registry is to identify risk factors or trends in the population. Barrett's esophagus (BE) represents the main pathological precursor to esophageal adenocarcinoma (EAC), a cancer that has increased in incidence more than any other cancer in the West over the last 30 years. ${ }^{3}$ The prevalence of $\mathrm{BE}$ in the US population may be as high as $5.6 \%$, and a UK single-center study of all upper gastrointestinal endoscopy found a frequency of $\mathrm{BE}$ of $1.6 \%$ in 1999 (having increased from $0.2 \%$ in 1977), implying that many patients with the condition 
remain undiagnosed. ${ }^{4-6}$ Barrett's registries have been established both in major academic centers, including the Cleveland Clinic and the Mayo Clinic, in whole populations, such as the Northern Ireland Barrett's Register (NIBR), and in regional collaborations, such as the UK Barrett's Registry ${ }^{1,6-10}$ Registry data have revealed many trends in epidemiology, including a decrease in mean age at diagnosis of Barrett's, and independent predictors for progression to adenocarcinoma, including male sex, older age, and low-grade dysplasia (LGD) at diagnosis. ${ }^{11-13}$ In patients with LGD-related Barrett's, the annual risk of EAC has been estimated at between $0.12-1.7 \%$, with lower predicted risks from whole population studies. LGD has a reported incidence of $8-9.8 \%$, and has a greater risk of disease progression than specialized intestinal metaplasia (SIM). However, there is considerable interobserver variability among pathologists with establishing a diagnosis of LGD. ${ }^{10,14}$

In Ireland, no population data on Barrett's registry exists. A registry was established in 2008 in St. James's Hospital, a center with specialist expertise in esophageal diseases, and we report herein the four-year experience of over a thousand patients at St. James' Hospital.

\section{METHODS}

A Barrett's registry was established in 2008 within an upper gastrointestinal medical-surgical group who established a protocol for patient eligibility, data collection, and protocols. A part-time (0.5 FTE, full time equivalent) data manager (MO'B) was appointed. Ethical approval was obtained. Data were collected retrospectively from charts of patients with pathologically confirmed $\mathrm{BE}$ attending the hospital for endoscopic surveillance, as well as all new diagnoses. BE was strictly identified by SIM in the esophageal biopsies. A data record form was prepared and completed for each patient entered into the registry based on their first visit and each subsequent visit; these data were entered into a computer database, initially using a PATS system in Microsoft Access, but in web format since 2011 (Dendrite, London, UK). Protocols were established for identifying Barrett's and performing Barrett's mapping based on extensive sampling, with recommendation of four-quadrant biopsies every $1 \mathrm{~cm}$.

Demographic information including age at diagnosis, smoking, and alcohol habits, weight, height, and body mass index (BMI), as well as symptoms, were computed. At endoscopy, the length of Barrett's segment and the presence of hiatus hernia were noted. Outcome of the endoscopy after the first and each subsequent visit were recorded including histological grade, and all treatments were recorded. All grades of dysplasia were categorized and verified by two independent specialist gastrointestinal pathologists. The patients were stratified according to the presence of LGD or indefinite for dysplasia on index endoscopy. Each patient was followed from date of diagnosis until the occurrence of one of the endpoints, or death, or emigration, to the end of December, 2012. Patients with an index diagnosis of high-grade dysplasia (HGD) totaled 60, 48 of whom were tertiary referrals, were all actively treated, and hence excluded from analysis of tumor progression.

\section{STATISTICS}

Data were presented as median and interquartile ranges. Incidence rates were analyzed for HGD, EAC, and combined EAC and HGD. Patients with HGD at index were excluded from this analysis. Patients were followed up until the occurrence of each endpoint or death. Cox-proportional hazard ratio was used to record relative risks of HGD and EAC according to the presence of LGD at baseline or in follow-up.

\section{RESULTS}

\section{Demographic data}

One thousand ninety-three patients were registered (Table 1). Sixty-seven percent of the patients were male, and the mean age was 56 years for men and 62 years for women $(P<0.05)$. Sixty-five percent of the patients were either current or former smokers, while $14 \%$ drank $>30$ units of alcohol/week. BMI was available in $47 \%$ of patients, and of these $31 \%$ were obese, and $73 \%$ were either overweight or obese. Over half $(51 \%)$ of the patients had evidence of a hiatus hernia. Nine hundred nineteen $(84 \%)$ of the patients were on proton pump inhibitors, and $89(8 \%)$ had undergone anti-reflux surgery. The most common symptoms reported prior to endoscopy and diagnosis were heartburn $(35 \%)$, dysphagia $(19 \%)$, epigastric pain $(13 \%)$, regurgitation $(7 \%)$, and vomiting $(6 \%)$. Seven percent of patients reported no symptoms.

\section{Endoscopic and pathological data}

The length of Barrett's segment was recorded from the index endoscopy. Where length was specified, short segment $\mathrm{BE}$ (defined as $<3 \mathrm{~cm}$ in length) was present in $38 \%$ of patients, while long segment Barrett's was present in $34 \%$ of patients, with no endoscopic Barrett's but SIM on histology only in $12 \%$ of patients. Thirty-seven percent of males had long-segment BE compared with $27 \%$ of females $(P<0.05)$.

(C) 2014 International Society for Diseases of the Esophagus 
Table 1 Characteristic of the 1093 patients with Barrett's esophagus

\begin{tabular}{|c|c|c|}
\hline Characteristic & \multicolumn{2}{|c|}{ Total cohort with Barrett's esophagus } \\
\hline \multicolumn{3}{|l|}{ Sex no. $(\%)$} \\
\hline Male & \multicolumn{2}{|l|}{$734(67.1 \%)$} \\
\hline Female & \multicolumn{2}{|l|}{$359(32.9 \%)$} \\
\hline \multicolumn{3}{|l|}{ Age at diagnosis of Barrett's esophagus - year } \\
\hline Median & \multicolumn{2}{|l|}{59} \\
\hline Interquartile range & \multicolumn{2}{|l|}{$49-69$} \\
\hline \multicolumn{3}{|l|}{ Person-year at risk } \\
\hline First year & \multicolumn{2}{|l|}{3599.83} \\
\hline \multirow[t]{2}{*}{$>1$ year } & \multicolumn{2}{|l|}{3532.40} \\
\hline & All & $>1$ year \\
\hline \multicolumn{3}{|l|}{ Time to diagnosis of adenocarcinoma - year } \\
\hline Median & 7.59 & 9.92 \\
\hline Interquartile range & $1.05-12.32$ & $6.41-14.04$ \\
\hline \multicolumn{3}{|l|}{ Age at diagnosis of adenocarcinoma - year } \\
\hline Median & 73.73 & 72.48 \\
\hline Interquartile range & $66.09-79.46$ & $67.44-78.73$ \\
\hline \multicolumn{3}{|l|}{ Time to diagnosis of high-grade dysplasia - year } \\
\hline Median & 1.57 & 3.28 \\
\hline Interquartile range & $0.5-3.5$ & $1.95-6.12$ \\
\hline \multicolumn{3}{|l|}{ Age at diagnosis of high-grade dysplasia/IMC - year } \\
\hline Median & 67.05 & 65.97 \\
\hline Interquartile range & $60.13-73.14$ & $56.92-72.88$ \\
\hline Total cases of esophageal adenocarcinoma in first year - no. & 3 & - \\
\hline Total cases of incident esophageal adenocarcinoma after first year - no. & 8 & - \\
\hline Total cases of high grade dysplasia in first year - no. & 15 & - \\
\hline Total cases of incident high grade dysplasia after first year - no. & 22 & - \\
\hline
\end{tabular}

IMC, intramucosal carcinoma.

On histological examination of biopsy specimens at the first endoscopic diagnosis, $83 \%$ of the patients had SIM only with no dysplasia evident, $3.8 \%$ were indefinite for dysplasia, 7.8\% had LGD, and 5.5\% had HGD. Of the men, $14.9 \%$ had LGD/HGD/EAC compared with $10.8 \%$ of women, and $6.9 \%$ of men had HGD/EAC compared with $2.6 \%$ of women $(P<0.05)$.

\section{Incidence of adenocarcinoma and high-grade dysplasia (Table 1)}

The total follow-up for all patients $(n=1045)$ was a median of 4.2 years (interquartile range 1.8 to 7.5 years) with a total follow-up of 3599 person-years. Eleven patients progressed to OAC, three in the first year, and eight thereafter. For HGD, and excluding 48 patients referred with HGD, or where EAC was present on initial endoscopy, 37 progressed, 15 in the first year and 22 beyond the first year. The overall incidence (\% per year) of EAC was 0.31 for invasive OAC and 1.03 for HGD (combined 1.33 combined, Table 2). After the exclusion of the 18 cases diagnosed during the first year of follow-up, the incidence of EAC was 0.23, HGD 0.62, and HGD/EAC combined was 0.85 . The incidence was higher in long segment compared with short-segment Barrett's $(P<0.001)$, and in patients $>60$ compared with those younger $(P<0.0001)$.

(C) 2014 International Society for Diseases of the Esophagus

\section{Implications of low-grade dysplasia at index endoscopy}

At the time of diagnosis, 85 patients $(7.7 \%)$ had a concurrent diagnosis of LGD. Of 73 with follow-up beyond one year, 46 had histological regression $(65 \%)$ to SIM at last visit, while eight progressed to HGD and six to EAC. Compared with SIM alone, the concurrent diagnosis increased the incidence beyond one year for HGD from 0.62 to 2.31, of EAC from 0.23 to 1.73 , and combined from 0.85 to 4 , respectively $(P<0.0001)$ (Figs 1,2$)$. Of this 85,33 were tertiary referrals, when excluded the incidence of HGD was 1.96, and 1.18 for EAC, 3.14 combined. When the first year was excluded, this was 1.19, 1.19, and 2.39, respectively. A small cohort of patients ( $n=$ 29) has pathology reports of 'indefinite for dysplasia', with an incidence rate to HGD of 4.36, all within the first year.

Sixty patients had HGD, and 18 underwent esophagectomy, 35 were treated with radiofrequency ablation, three regressed to LGD, and the remaining continue on surveillance.

\section{DISCUSSION}

Esophageal adenocarcinoma has increased markedly in the West, in the United Kingdom, for instance, it is predicted to increase by a further $40 \%$ by $2020 .^{3,4,15}$ 
Table 2 All follow-up data

\begin{tabular}{|c|c|c|c|c|c|c|c|}
\hline Characteristic & $\begin{array}{l}\text { Patients } \\
\text { no. }\end{array}$ & $\begin{array}{l}\text { Person- } \\
\text { years }\end{array}$ & $\begin{array}{l}\text { High-grade } \\
\text { dysplasia } \\
\text { HGD) No. }\end{array}$ & $\begin{array}{l}\text { Adenocarcinoma } \\
\text { no. }\end{array}$ & $\begin{array}{l}\text { Incidence of high } \\
\text { grade dysplasia } \\
\% \text { per year }\end{array}$ & $\begin{array}{l}\text { Incidence of } \\
\text { adenocarcinoma } \\
\% \text { per year }\end{array}$ & $\begin{array}{l}\text { Incidence of HGD/ } \\
\text { adenocarcinoma } \\
\% \text { per year }\end{array}$ \\
\hline Total & 1093 & 3684.83 & 37 & 11 & 1.00 & 0.30 & 1.30 \\
\hline \multicolumn{8}{|l|}{ Sex } \\
\hline Female & 359 & 1128.45 & 11 & 1 & 0.97 & 0.09 & 1.06 \\
\hline Male & 634 & 2556.38 & 26 & 10 & 1.02 & 0.39 & 1.41 \\
\hline \multicolumn{8}{|l|}{ Age, year } \\
\hline$<50$ & 297 & 1296 & 4 & 1 & 0.31 & 0.08 & 0.39 \\
\hline $50-59$ & 256 & 1085.75 & 6 & 0 & 0.55 & 0.00 & 0.55 \\
\hline $60-69$ & 284 & 837.2 & 14 & 3 & 1.67 & 0.36 & 2.03 \\
\hline $70-79$ & 191 & 386.66 & 11 & 4 & 2.84 & 1.03 & 3.88 \\
\hline$\geq 80$ & 65 & 79.22 & 2 & 3 & 2.52 & 3.79 & 6.31 \\
\hline \multicolumn{8}{|c|}{ Length of Barrett's esophagus segment } \\
\hline Short & 414 & 1045.72 & 9 & 0 & 0.86 & 0.00 & 0.86 \\
\hline Long & 369 & 1276.81 & 17 & 6 & 1.33 & 0.47 & 1.80 \\
\hline Unknown & 176 & 818.73 & 9 & 5 & 1.10 & 0.61 & 1.71 \\
\hline Histology only & 134 & 543.57 & 2 & 0 & 0.37 & 0.00 & 0.37 \\
\hline \multicolumn{8}{|c|}{ Dysplasia at index biopsy } \\
\hline No & 931 & 3199.78 & 19 & 4 & 0.59 & 0.13 & 0.72 \\
\hline Indefinite & 29 & 45.93 & 2 & 0 & 4.35 & 0.00 & 4.35 \\
\hline Low-grade dysplasia & 85 & 354.12 & 16 & 7 & 4.52 & 1.98 & 6.49 \\
\hline HGD & 60 & - & - & - & $\mathrm{n} / \mathrm{a}$ & $\mathrm{n} / \mathrm{a}$ & $\mathrm{n} / \mathrm{a}$ \\
\hline \multicolumn{8}{|l|}{ Smoking status } \\
\hline Never smoked & 296 & 1186.39 & 14 & 2 & 1.18 & 0.17 & 1.35 \\
\hline Ex-smoker & 356 & 1170.18 & 13 & 6 & 1.11 & 0.51 & 1.62 \\
\hline Current smoker & 205 & 750.26 & 5 & 1 & 0.67 & 0.13 & 0.80 \\
\hline Unknown & 236 & 578 & 5 & 2 & 0.87 & 0.35 & 1.21 \\
\hline \multicolumn{8}{|l|}{ Alcohol intake } \\
\hline Nil & 216 & 842.14 & 14 & 3 & 1.66 & 0.36 & 2.02 \\
\hline $0-30$ units & 449 & 1637.73 & 14 & 5 & 0.85 & 0.31 & 1.16 \\
\hline$>30$ units & 108 & 413.94 & 1 & 0 & 0.24 & 0.00 & 0.24 \\
\hline Unknown & 320 & 791.02 & 8 & 3 & 1.01 & 0.38 & 1.39 \\
\hline \multicolumn{8}{|l|}{ BMI } \\
\hline$<30$ & 360 & 1435.5 & 16 & 7 & 1.11 & 0.49 & 1.60 \\
\hline$>30$ & 161 & 677.72 & 10 & 1 & 1.48 & 0.15 & 1.62 \\
\hline Unknown & 572 & 1571.61 & 11 & 3 & 0.70 & 0.19 & 0.89 \\
\hline
\end{tabular}

Barrett's esophagus is also increasing in incidence, and a $40 \%$ increase in the incidence of BE in men and a 17\% increase in women were reported between 1992 and 2003 in the Netherlands. ${ }^{15}$ Barrett's registries have successfully been set up in many countries including in Northern Ireland (NIRB) and the United Kingdom (UKBOR), and population data on Barrett's has been abstracted from national pathology and cancer registries.,16 A Barrett's registry provides enormous potential for managing patients

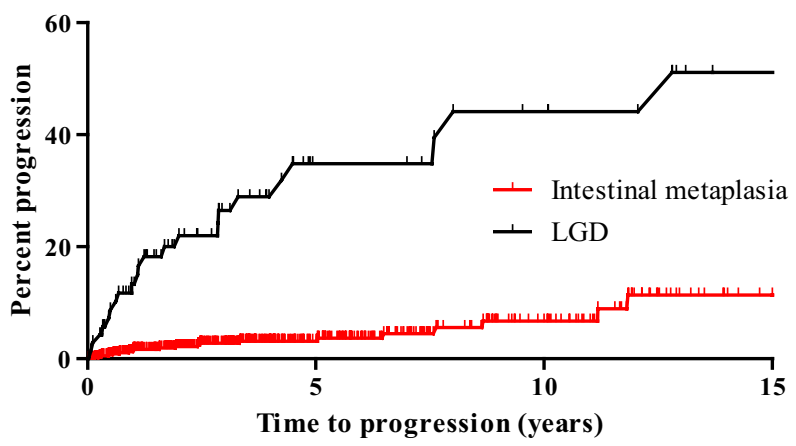

Fig. 1 Progression to high-grade dysplasia (HGD) and esophageal adenocarcinoma (EAC). with Barrett's, and for clinical and scientific research which is increasingly focused on identifying populations at greatest risk and hence in need of targeted therapy and close surveillance. This is particularly important where Barrett's surveillance for all is not cost-effective. ${ }^{17}$ Initial experience from one center, a proposed potential template for a national registry, highlights several factors. First, the presence of dysplasia at index biopsy is approximately $13 \%$; the annual risk of progression, at 0.85 , is higher than

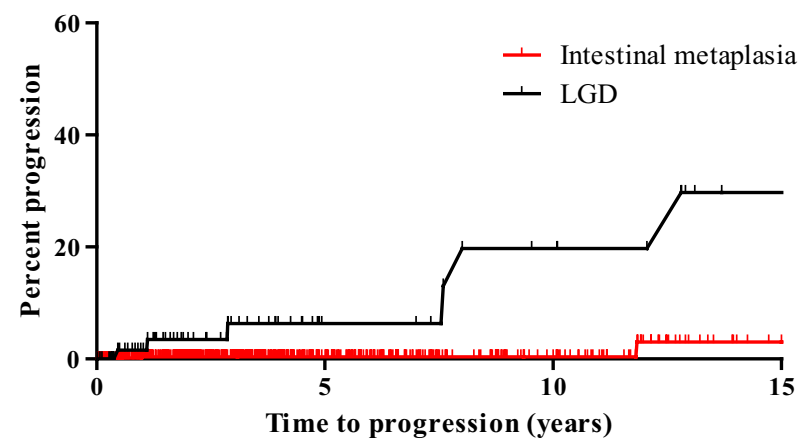

Fig. 2 Progression to esophageal adenocarcinoma (EAC) only. (C) 2014 International Society for Diseases of the Esophagus 
recently reported population studies; and LGD at index biopsy represents an approximate six to eight-fold increased risk of progression compared with SIM alone.

The approximate 2: 1 male-to-female ratio of Barrett's in this population is consistent with other reports. ${ }^{8,16}$ Lifestyle factors are postulated to associate with Barrett's population, although the data are inconsistent. A recent study has shown increased risk of HGD and EAC development in smokers with BE ${ }^{17}$ Obesity is linked to EAC, possibly through increased reflux and hiatal herniae, but whether obesity causes SIM or disease progression remains controversial. ${ }^{18-20}$ In this series, $31 \%$ of patients were obese, and $76 \%$ obese or overweight, and although this is higher than population norms, it is similar to data from cohorts with GERD and no Barrett's from a previous Irish study. ${ }^{19,21}$ The relationship between obesity, Barrett's, and EAC requires further study, both clinical and scientific.

It is uncertain whether the length of Barrett's changes is associated with cancer risk. ${ }^{12,22}$ It is also reported that that the length of columnar-lined esophagus does not change over time, and even if it does change, this does not affect the risk of carcinogenesis; therefore it is important to note the length of segment at initial diagnostic endoscopy. In this series, 37\% of men had long-segment SIM, compared with $27 \%$ of women, and long-segment SIM was associated with an incidence of progression of $1.89 \%$ per year, compared with $0.88 \%$ for shortsegment SIM.

The principal finding in follow-up of this population was that the absolute risk of EAC was higher than recently reported in whole population studies. Compared with a Danish population study of 11028 patients, and a Northern Ireland Barrett's Register (NIBR) study of 8522 patients, the incidence of HGD and EAC was higher. ${ }^{8,16}$ Excluding cases observed in the first year, which may indicate prevalent changes, the incidence of HGD was 0.62 in this study compared with 0.19 and 0.09 in the Danish and NIBR studies, respectively. The incidence of EAC was 0.23 , 0.12 , and 0.13 in the current, Danish, and NIBR studies, respectively. The combined rates of HGD/ EAC were accordingly between four and five times higher than the Danish and NIBR study. This is consistent with other studies of similar size and follow-up. ${ }^{910,23,24}$ It is possible that larger population registries are less prone to referral or diagnostic basis, and our own series, although comprehensive and nonselected, and where tertiary cases were excluded, may be biased toward generally higher risk patients in view of the hospital being a tertiary center for esophageal diseases. Conversely, in this center, as opposed to population studies, it is possible that a more rigorous biopsy protocol applies, with increased awareness among endoscopists and pathologists, with consequent higher incident rates. A reasonable inference from comparing this study to these population studies may be that caution should apply to accepting an actual annual risk of $0.12 \%$ (Danish), and $0.22 \%$ (NIBR) applies to Barrett's populations diagnosed and managed in specialist centers. ${ }^{8,16}$

In this study, the criterion for a diagnosis of Barrett's esophagus was SIM, this is similar to the Danish study and to current standards in many countries, including North America. ${ }^{16}$ In the United Kingdom, SIM with the identification of goblet cells is not a prerequisite, and columnar lined epithelium (CLE) as was used in the NIBR studies, suffices. ${ }^{8,9}$ Some associations between CLE and EAC exist, although it has been suggested that the lack of SIM may simply reflect sampling technique or pathologist misclassification. Notwithstanding this difference, the exclusion of CLE without SIM from our register may also slightly increase incidence rates. With respect to initial pathology, the presence of LGD at index biopsy, detected in $7.9 \%$ of our population, is the key finding demanding close surveillance. Previous studies have shown that LGD has a relative risk of 2.871-5.5 for development of EAC compared with non-dysplastic Barrett's epithelium. ${ }^{5,25}$ In the NIBR study, the incidence rate of progression was $1.4 \%$ per year compared with $0.17 \%$ for no dysplasia, an adjusted hazards ratio of 5.7. In this study, the incidence rates of HGD and EAC was markedly increased in patients with LGD at baseline compared with those that did not, $6.5 \%$ compared with $0.72 \%$ for all, and $4 \%$ compared with $0.5 \%$ if the first year is excluded. Even excluding 33 patients with LGD who were tertiary referrals, $3.14 \%$ compared with $0.72 \%$ with SIM alone progressed, and 2.39 compared with 0.78 if the first year was excluded. A similar relative risk of 4.8 was observed in the Danish study. ${ }^{16}$ Only $3.9 \%$ of patients with SIM in initial histology progressed to HGD/EAC; however in LGD patients, $32.3 \%$ progressed to $\mathrm{HGD} / \mathrm{EAC}$, consistent with recent series. ${ }^{26}$ Although the cost-effectiveness of regular surveillance for a cancer risk less than $0.5 \%$ is well accepted, the annual risk of progression associated with LGD on index biopsy establishes this as probably cost-effective, as $36 \%$ of incident HGD and $75 \%$ EAC were within this group.

In conclusion, this study demonstrates the feasibility and usefulness of a large Barrett's registry in a tertiary center. Four centers combined in 2011 to form a larger collaborative registry using this prospective web-based database, and this will hopefully evolve into a national registry. The data suggest that the recently reported population registry data from Northern Ireland and Denmark will underestimate risk at a specialist center, even where tertiary referrals are excluded. Low-grade dysplasia at index biopsy represents a significant risk of progression and demands close surveillance. It is hoped and 
anticipated that future larger scale projects in Ireland, through the national network, will establish solid prospective data on large populations and underpin clinical and scientific research both nationally and through international collaboration.

\section{References}

1 Gatenby P A C, Caygill P J, Watson A, Murray L, Romero Y. Barrett's esophagus registries. Ann N Y Acad Sci 2011; 1232: 405-10.

2 Barrett G, Cassell J A, Peacock J L, Coleman M P. National survey of British public's views on use of identifiable medical data by the National Cancer Registry. BMJ 2006; 332: 1068 72.

3 Cook M B, Chow W H, Devesa S S. Oesophageal cancer incidence in the United States by race, sex and histologic type. $\mathrm{Br}$ J Cancer 2009; 101: 855-9.

4 Steevens J, Botterweck A A, Dirx M J, van der Brandt P A, Schouten L J. Trends in incidence of oesophageal and stomach cancer subtypes in Europe. Eur J Gastroenterol Hepatol 2010; 22: 669-78.

5 Caygill C P, Reed P I, Johnston B J, Hill M J, Ali M H, Levi S. A single centre's 20 years' experience of columnar-lined (Barrett's) oesophagus diagnosis. Eur J Gastroenterol Hepatol 1999; 11: 1355-8.

6 Ramus J R, Gatenby P A C, Caygill C P J, Winslet M C, Watson A. Surveillance of Barrett's columnar-lined oesophagus in the UK: endoscopic intervals and frequency of detection of dysplasia. Eur J Gastroenterol Hepatol 2009; 21: 636-41.

7 O'Connor J B, O'Connor J B, Falk G W, Richter J E. The incidence of adenocarcinoma and dysplasia in Barrett's esophagus: report on the Cleveland Clinic Barrett's Esophagus Registry. Am J Gastroenterol 1999; 94: 2037-42.

8 Bhat S, Coleman H G, Yousef Y et al. Risks of malignant progression in Barrett's esophagus patients: results from a large population-based register. J Natl Cancer Inst 2011; 103: 1-9.

9 Murray L, Watson P, Johnston B et al. Risk of adenocarcinoma in Barrett's oesophagus: population based study. BMJ 2003; 327: 534-5.

10 de Johnge P J, van Blankenstein M, Looman C W, Casparie M K, Meijer G A, Kuipers E J. Risk of malignant progression in patient with Barrett's oesophagus. A Dutch nationwide cohort study. Gut 2010; 59: 1030-6.

11 Thomas T, Abrams K R, De Caestrecker J S, Robinson R J. Meta analysis: cancer risk in Barrett's oesophagus. Aliment Pharmacol Ther 2007; 26: 1465-77.

12 Youssef F, Caldwell C, Cantwell M M, Galway K, Johnston B T, Murray L. The incidence of esophageal cancer and high-grade dysplasia in Barrett's esophagus: a systematic review and meta-analysis. Am J Epidemiol 2008; 168: 237-49.

13 Sikkema M, de Yonge P J F, Steyerbergrg E W, Kuipers E J. Risk of esophageal adenocarcinoma and mortality in patients with Barrett's esophagus: a systematic review and metaanalysis. Clin Gastroenterol Hepatol 2010; 8: 235-44.

14 Lagergren J. Adenocarcinoma of oesophagus: what exactly is the size of the problem and who is at risk? Gut 2005; 54 (Suppl 1): i1-i5.

15 Post P N, Siersema P D, Van Dekken H. Rising incidence of clinically evident Barrett's oesophagus in The Netherlands: a nation-wide registry of pathology reports. Scand J Gastroenterol 2007; 42: 17-22.

16 Hvid-Jensen F, Pederson L, Drewes A M, Sorensen H T, Fuch-Jensen P. Incidence of adenocarcinoma among patients with Barrett's esophagus. N Engl J Med 2011; 365: 1375-83.

17 Provenzale D, Schmidt C, Wong J B. Barrett's esophagus: a new look at surveillance based on emerging estimates of cancer risk. Am J Gastroenterol 1999; 94: 2043-53.

18 Coleman H G, Bhat S, Johnston B T, McManus D, Gavin A T, Murray L J. Tobacco smoking increases the risk of high-grade dysplasia and cancer among patients with Barrett's esophagus. Gastroenterology 2012; 142: 233-40.

19 Caygill C P, Johnston D A, Lopez M et al. Lifestyle factors and Barrett's esophagus. Am J Gastroenterol 2002; 97: 1328-31.

20 Abnet C C, Freedman N D, Hollenbeck A R, Fraumeni J F Jr, Leitzmann M, Schatzkin A. A prospective study of BMI and risk of oesophageal and gastric adenocarcinoma. Eur J Cancer 2008; 44: 465-71.

21 Healy L A, Ryan A M, Pidgeon G, Ravi N, Reynolds J V. Lack of differential pattern in central adiposity and metabolic syndrome in Barrett's esophagus and gastroesophageal reflux disease. Dis Esophagus 2010; 23: 386-91.

22 Gatenby P A, Caygill C P, Ramus J R, Charlett A, Fitzgerald R C, Watson A. Short segment columnar-lined oesophagus: an underestimated cancer risk? A large cohort study of the relationship between Barrett's columnar-lined oesophagus segment length and adenoacarcinoma risk. Eur J Gastroenterol Hepatol 2007; 19: 969-75.

23 Sharma P, Falk G W, Weston A P, Reker D, Johnston M, Sampliner R E. Dysplasia and cancer in a large multicenter cohort of patients with Barrett's esophagus. Clin Gastroenterol Hepatol 2006; 4: 566-72.

24 Solaymani-Dodaran M, Logan R F, West J, Card T, Copeland C. Risk of oesophageal cancer in Barrett's oesophagus and gastro-oesophageal reflux. Gut 2004; 53: 1070-4.

25 Oberg S, Wenner J, Johansson J, Walther B, Willén R. Barrett esophagus: risk factors for progression to dysplasia and adenocarcinoma. Ann Surg 2005; 242: 49-54.

26 Lim C H, Treanor D, Dixon M F, Axon A T. Low-grade dysplasia in Barrett's esophagus has a high rate of progression. Endoscopy 2007; 39: 581-7. 